\title{
Protocol for the adaptation of clinical practice guidelines for the management of physical restraints in critically ill patients
}

\author{
Nianqi Cui ${ }^{1}$, Yuping Zhang ${ }^{1}$, Yuanfei Liu ${ }^{1}$, Yingfeng Zhou ${ }^{2}$, Hong Sun ${ }^{3}$, Jingfen Jin ${ }^{1,4}$; on behalf of the \\ guideline adaptation working group of physical restraints \\ ${ }^{1}$ The Second Affiliated Hospital Zhejiang University School of Medicine (SAHZU), Hangzhou, China; ${ }^{2}$ School of Nursing, Fudan University, \\ Shanghai, China; ${ }^{3}$ Peking Union Medical College Hospital, Beijing, China; ${ }^{4}$ Changxing Branch Hospital of SAHZU, Huzhou, China \\ Correspondence to: Jingfen Jin, MHA, RN. Professor, Assistant Dean, Executive President, The Second Affiliated Hospital Zhejiang University School \\ of Medicine (SAHZU), Hangzhou 310009, China; Changxing Branch Hospital of SAHZU, Huzhou 313100, China. Email: zrjzkhl@zju.edu.cn.
}

\begin{abstract}
Background: The effectiveness of physical restraints (PRs) in the prevention of unplanned extubation has been increasingly questioned, and several countries have developed clinical practice guidelines for reducing the use of PRs. However, some countries, including China, have failed to establish their guidelines, and the adaptation of existing guidelines to local circumstances may be an attractive alternative.

Methods: Following the guideline definition of Institute of Medicine, the guideline will be adapted following the CAN-IMPLEMENT@. Guideline scope and clinical questions will be established based on an integrative review, retrospective study, and stakeholder interviews. The guideline's quality will be assessed by the Appraisal of Guidelines for Research and Evaluation II and Appraisal of Guidelines Research and Evaluation-Recommendations Excellence. A systematic review and meta-analysis will be evaluated by A Measurement Tool to Assess Systematic Reviews. The guidelines will meet the criteria of the RIGHT-Ad@ pt Checklist.

Discussion: This study describes the proposed protocol for adapting clinical practice guidelines on PRs in critically ill patients. We believe the guideline will help health professionals, especially critical care nurses, deliver safe, high-quality patient care by reducing the implementation of PRs in China.

Guideline Registration: The guideline has been registered at the International Practice Guidelines Registry Platform (http://www.guidelinesregistry.org/). The registration number is IPGRP-2019CN094, registration date 27-Dec-2019.
\end{abstract}

Keywords: Clinical practice guideline; physical restraints (PRs); intensive care units (ICUs); guideline adaptation; CAN-IMPLEMENT@

Submitted Oct 29, 2020. Accepted for publication Jan 21, 2021.

doi: 10.21037/apm-20-2133

View this article at: http://dx.doi.org/10.21037/apm-20-2133

\section{Introduction}

The definition of physical restraints (PRs) is "Any action or procedure that prevents a person's free body movement to a position of choice and/or normal access to his/her body by the use of any method, attached or adjacent to a person's body that he/she cannot control or remove easily" (1). PRs vary greatly in different countries, but the utilization rate of PRs in intensive care units (ICUs) is generally higher than in general wards. One study indicated that the implementation rate of PRs in ICUs is 23.4 times that of general wards (2). Another, involving 68 ICUs in the United States showed that the rate of PRs in ICUs was 33\% (3). A recent multicenter cross-sectional study conducted in China has shown that PRs use in ICUs was 59.07\% (4).

The most common reason for the use of PRs in ICUs is to prevent unplanned extubation $(5,6)$. The results of a four-year quality improvement program showed that the use of PRs in ICU among patients with agitation increased from $58 \%$ to $90 \%$, and the incidence of unplanned extubation 
decreased from $10 \%$ to $4 \%$ (7). Based on their clinical experience, critical care nurses indicated that PRs could effectively prevent unplanned extubation $(8,9)$. However, there has been no randomized controlled trial study investigating the effectiveness and safety of PRs (10). On the contrary, some studies have found that PRs do not prevent UE $(11,12)$ and increase its incidence and the likelihood of reintubation $(12,13)$. The related complications caused by PRs include neurovascular complications, such as edema and mobility impairment of restrained limbs (14), an increased incidence of pressure ulcers (15), prolonged length of hospital stay (12), and increased use of benzodiazepines, opioids, and antipsychotics (16), which can incite greater agitation in restrained patients (16). At the same time, PRs are a risk factor for delirium $(17,18)$ and may also increase the incidence of post-traumatic stress disorder after ICU discharge (18), emotional disorders (19), depression, body image disturbance, social isolation, dignity loss, and decline of perceptual and sensory abilities (20). As the effectiveness of PRs for patient safety has been questioned, several countries have developed clinical practice guidelines (CPGs) aimed at standardizing the management of PRs and decreasing their use.

In 2012, the Registered Nurses' Association of Ontario released a CPG titled: Promoting safety: alternative approaches to the use of restraints (21). The guideline focuses on alternative approaches and provides 12 recommendations from three main aspects: practice, education, organization, and policy. The recommendations concerning practice are as follows: To establish a therapeutic relationship between nurses and patients, nurses should understand the behaviors of patients at risk of self or others injury and avoid PRs; nurses need to use validated tools to determine if PRs are necessary for the patient; to reduce PRs, multi-component strategies and interprofessional teams are necessary. The recommendations concerning education include nurses' education to increase their knowledge and change their attitude and behavior towards the implementation of PRs. Changes to an organizational culture that ensure patient rights and staff safety through the implementation of risk management and quality improvement and powerful health resources and organizational support are necessary for implementing guidelines minimizing the use of restraints. In 2014, The University of Iowa released the guideline: Changing the Practice of Physical Restraint Use in Acute Care, which was an update of a previous version and based on best available evidence (22). The guideline is principally directed at nurse-driven approaches to reducing restraints, and the recommendations include physical, physiological, and environmental changes. It is worth mentioning that the recommendations are presented with a flow chart, which is conducive to the clinical implementation of the guideline. The Society of Critical Care Medicine released the Clinical Practice Guidelines for the Prevention and Management of Pain, Agitation/Sedation, Delirium, Immobility, and Sleep Disruption in Adult Patients in the ICU in 2018 (10), which is an update and expansion of the CPGs of 2013 (23). Of the 37 recommendations, two topics, rehabilitation/ mobilization, and sleep were added to the update. While there were no direct recommendations for the management of PRs, ungraded statements about PRs were included in the agitation/sedation section and mainly concerned with the rationale and evidence gaps. However, the clinical practice of PRs is closely related to the management of pain, agitation/sedation, and delirium, and the guidelines have implications for the practice of PRs in ICUs on this basis.

There are currently no CPGs on PRs in China. The development of new CPGs is time-consuming and requires considerable expertise (24). Guideline adaptation is the systematic approach to the endorsement and/or modification of a guideline(s) produced in one cultural and organizational setting for application in a different context (25). Given the existence of high-quality guidelines, adaptation may be the alternative to developing new, locally relevant guidelines. We aim to adapt existing guidelines on PRs in ICUs to apply these in the Chinese context.

\section{Methods}

The process of developing the guideline will follow the 4.0 version of CAN-IMPLEMENT $\odot$, a method for guideline adaptation and implementation, which was initially developed to assist groups to adapt cancer care guidelines originally developed outside of their jurisdiction (26). Adaptation will occur in five steps based on CAN-IMPLEMENTC: Call to action, Guideline Development Plan, Search \& Screen, Assess \& Select, Draft, Revise \& Endorse. The purpose of the Call to action step is to clarify the driving forces of the guideline adaptation and the organizational context. The next step. Guideline Development Plan will solve objectives, including establishing the guideline scope, the working panel, and a detailed adaptation plan. Following this, locating relevant guidelines and evidence will be the primary tasks in the Search \& Screen step. After that, the evidence will be appraised in the Assess \& Select step, and recommendations will be achieved by consensus. The 
final step is Draft, Revise \& Endorse, where a draft adapted guideline will be prepared for external review and endorsed by target users.

\section{Form steering committee and working panels}

\section{The Guideline Steering Committee}

The steering committee will include 15 multidisciplinary experts: intensivists, emergency physicians, critical care nurses, emergency nurses, a health policy-maker/quality management researcher, a methodologist, a reference librarian, a patient with a history of PRs in ICU, and a family member. The steering committee's role is to establish other working groups, manage conflicts of interest, review the guideline scope and clinical questions, approve the work plan, supervise the guideline adaptation process, review the final guideline document, and provide necessary consultation and guidance for the adaptation of the guideline.

\section{The Guideline Adaptation Group}

The guideline adaptation group role will be to follow the World Health Organization handbook (27) and the role of guideline adaptation group established by Chen et al. (28). All members will sign a conflict of interest statement, which will be reported in the final guideline. This guideline's adaptation is supported by the Science Research Foundation of Chinese Nursing Association (grant numbers: ZHKY201913) and Zhejiang University Academic Award for Outstanding Doctoral Candidates (grant numbers: 202059). The funders have no role in the adaptation process.

\section{The chair of the Guideline Adaptation Group}

The chair of the guideline adaptation group will include one chairman and two vice-chairmen. The chairman is also the chairman of the emergency nursing committee of Chinese Nursing Association. One vice chairman is the chairman of the intensive care committee of Chinese Nursing Association, and the other is a methodologist. The chairman and one vice-chairman will be experts in facilitating groups that reach decisions based on consensus and be experienced at critically appraising and interpreting evidence and at developing evidence-informed recommendations. The methodologist will provide methodological guidance, supervise the guideline's adaptation process, and be responsible for the quality of the guideline.

\section{The Secretary Group}

The secretary group will include one Ph.D. student whose research direction is critical care, a postgraduate, and a nursing department staff member. The secretary group's responsibilities are coordinating the work of other working groups, writing the adaptation work plan, collecting the guideline scope and clinical questions, organizing and recording guideline meetings, and drafting and submitting the guideline.

\section{The Evidence Evaluation Group}

The evidence evaluation group will include a reference librarian, one Ph.D. student, and three nurses with an evidence-based nursing research background. The evidence evaluation group will search, screen, record, and assess the guidelines and systematic review (SR)/meta-analysis, complete the developing the recommendations matrix and develop the recommendations matrix.

\section{The Consensus Group}

The consensus group will consist of 30 multidisciplinary experts, including experts in intensive care medicine, emergency medicine, critical care, emergency nursing, and a patient with a history of PRs in ICU and her/his family member from the eastern, western, central, and northeast of China. The consensus group's main responsibilities will be to participate in the modified Delphi inquiry, reach a consensus, and approve the guideline.

\section{The External Review Group}

The external review group will comprise experts in intensive care medicine, critical care, health policy, quality management, methodology, and a patient with a history of PRs in ICU and her/his family member. The external review group's primary role will be to review the final guideline and ensure the scientificity, clarity, and equality of the final guideline.

\section{Evidence search}

An evidence search based on the results of an integrative review, historical data analysis, and qualitative descriptive study will be carried out according to the guideline scope and the clinical questions in Dec 2020. The integrative review will follow the methodology presented by Whittemore and Knafl (29), and potential evidencebased practice initiatives about the management of PRs 
will emerge using this approach. Historical data will be extracted in the implementation site and analyzed to outline real practice problems. The current state of PRs practice and factors influencing critical care clinicians' decisions to implement PRs in ICUs will be obtained through one-toone, semi-structured interviews with critical care nurses and intensivists, following a qualitative descriptive design. The guideline's scope will include the management of PRs, unplanned extubation, mechanical ventilation, pain, agitation/sedation, delirium, and visiting/family engagement in ICUs. PIPOH (P-Population, I-Intervention, $\mathrm{P}$-Professionals/Patients, O-outcomes, H-Healthcare Setting) will be used to describe the clinical questions. The guideline is mainly targeted for critical care nurses in ICUs, and the target and benefit population are critically ill patients in ICUs. Outcomes will include patient outcomes (the incidence of PRs, unplanned extubation, delirium), professional outcomes (knowledge, attitudes, perception about PRs), and system outcomes (practice variation).

The guidelines on cross-database search platform, professional associations, and databases will be searched (Table S1). General searching will be conducted to retrieve the guidelines on cross-database search platform and professional associations, and advanced searches will be conducted to retrieve guidelines on databases. The guideline's scope will develop key concepts, and the search strategy will include synonyms, alternate spellings, and truncation of the key concepts developed by the evidence evaluation group. An example of the cross-database search platform concerning guidelines and an example of the databases used are shown in Table S2.

Screening decisions will follow the CANIMPLEMENT@ flow chart. Two reviewers in the evidence evaluation group will follow the inclusion and exclusion criteria to screen the guidelines/SR/metaanalysis. The inclusion criteria will be: language: English/ Chinese; publication type: guidelines/SR/meta-analysis; and publication date Jan-2011 to Dec-2020 for guidelines and Jan-2016 to Dec-2020 for SR/meta-analysis. The guideline definition will follow the definition of Institute of Medicine: Clinical guidelines are statements that include recommendations intended to optimize patient care that are informed by a SR of evidence and an assessment of the benefits and harms of alternative care options (30). The National Guideline Clearinghouse formally adopted the definition in 2014. Therefore, if the guideline was developed before 2014, the definition will follow the Institute of Medicine of 1990: Clinical practice guidelines are systematically developed statements to assist practitioners and patient decisions about appropriate health care for specific circumstances (31); at the same time, the guidelines should include three characteristics: Scientific or research evidence integration, an explanatory summary of the evidence, and clear recommendations based on evidence (31). The exclusion criteria will be: guidelines fail to meet definition criteria or guideline scope; the healthcare setting of the guidelines/SR/meta-analysis are only for patients in mental health centers, nursing homes, and home; translation version, simplified version, historical version, interpretation or explanations, review, appraisal methodology or implementation of the guidelines; the target and benefit population are neonates or children (age $<18$ ); guidelines/SR/meta-analysis are only for pharmacological intervention or treatment of specific disease.

\section{Evidence evaluation and selection}

Rigour of the development: five members will use AGREE II (Appraisal of Guidelines for Research and Evaluation II) to assess the included guidelines' quality. Combined with domain ratings, a minimum methodological threshold for recommended guidelines is $60 \%$ of 6 domains, and the recommended guidelines with modifications is a minimum of $30 \%$ of 3 domains but less than $60 \%$. Timeliness: the evidence evaluation group will review the publication date and update the guidelines to determine whether the latest evidence has been included. If the guidelines' quality is high, but the evidence description has not yet been updated, the evidence description will be updated by the secretary group through the evidence obtained from the $\mathrm{SR} /$ meta-analysis. Clinical applicability, values, preferences, and implementability: Even if the guidelines meet the methodological requirements, the recommendations' credibility or implement ability cannot be guaranteed. Therefore, it is necessary to evaluate the quality of the recommendations of the guidelines using the AGREEREX (Appraisal of Guidelines Research and EvaluationRecommendations Excellence) (32). Guidelines will be divided into high quality (overall scores $>70 \%$ ), lower quality (overall scores $<30 \%$ ), and moderate quality. A guideline content analysis and assessment matrix will describe the results of evidence evaluation and selection of the included guidelines (33) (Table S3). 
The SR/meta-analysis quality will be assessed by the AMSTAR 2 (A Measurement Tool to Assess Systematic Reviews). Although the AMSTAR 2 consists of 16 items, critical domains include items $2,4,7,9,11,13$, and 15 . The guidelines will select high-quality SR/meta-analysis, which means at most, one critical domain indicates weakness (34). Two members will use AMSTAR 2 [Chinese version (35)] to assess the quality of the included SR/meta-analysis and then discuss with a third reviewer to achieve consensus in case of disagreements. Content analysis and appraisal of the SR/ meta-analysis matrix are shown in Table S4.

\section{Formulate recommendation and reach consensus}

The consensus group will reach a consensus for recommendations through a modified Delphi (36), developed by the American Society of Clinical Oncology (37). The consensus group experts will evaluate benefits \& harms of the options, quality of the evidence, resources required, cost-effectiveness, equality, feasibility, values, and preferences, and acceptability to give recommendations. Benefit \& harms of the options and quality of the evidence will be sourced from the quality assessment process. Values, preferences, and acceptability will be conducted by a crosssectional survey of stakeholders in eastern, central, western, and northeast China about the recommendations, which offer experts' reference evidence. Resources required, costeffectiveness, equality, and feasibility will be evaluated through the experience of experts. The recommendation matrix is shown in Table S5.

\section{Draft, revise and endorse recommendations}

The secretary group will prepare the first draft of the guideline for internal and external review. The guideline steering committee will complete the internal review through PANELVIEW (38). The external review group will use AGREE II and AGREE-REX to conduct an external review.

\section{Prepare the final guideline and establish a renewal plan}

The final guideline content will follow the CANIMPLEMENT template (26) and meet the criteria of the RIGHT-Ad@pt Checklist (39). Updates of the guideline will be determined by updated evidence, and the guideline may be terminated or modified $(40,41)$. It is planned to update the guideline every 5 years.

\section{Guideline adapters}

The Emergency Nursing Committee of Chinese Nursing Association, Intensive Care Committee of Chinese Nursing Association, The Second Affiliated Hospital of Zhejiang University School of Medicine, and Fudan University Cooperation Center of Joanna Briggs Institute EvidenceBased Nursing are the adapters for the guideline adaptation.

\section{Guideline registration}

The guideline has been registered at the International Practice Guidelines Registry Platform (http://www. guidelines-registry.cn/index). The registration number is IPGRP-2019CN094.

\section{Discussion}

There are no guidelines about PRs on critically ill patients in China to the best of our knowledge. This is the first study to adapt high-quality guidelines about PRs for local use based on CAN-IMPLEMENT@.

Gaps still exist between evidence and practice (42). Although much evidence has proven the negative impact of PRs on patients, PRs are still commonly used in China. A recent multicenter cross-sectional study conducted showed that PRs use in ICUs was 59.07\% in China (4). The International Council of Nurses called for evidencebased practice among nurses worldwide to close the gap (43). CPGs bridge the gap between evidence and practice and are important tools for health professionals aspiring to implement evidence into practice (44). The lack of local restraint guidelines is one of the barriers to reducing PRs (45); therefore, it is necessary to develop guidelines concerning their use (46). Although CPGs can help health professionals in clinical decision-making processes and reduce variability in clinical practice, the development of guidelines requires a wealth of resources (44). Guideline adaptation has been proposed as an alternative to developing new guidelines where high-quality guidelines are already available (44). CAN-IMPLEMENT@ is a guideline adaptation and implementation planning resource that was initially developed to assist groups in adapting cancer care guidelines. The implementation planning perspective and facilitation aspects have been expanded, and an epilogue on the Joanna Briggs Institute framework has been added to incorporate an international viewpoint (26). We believe the guideline adapted by CAN-IMPLEMENT@ will guide health professionals, especially critical care nurses, in the decision-making process about PRs in ICUs. 
However, there are also several limitations to our study. Due to resource constraints, we will only search for English and Chinese guidelines and SR/meta-analysis. Also, because high-quality guidelines about PRs are limited, we will not follow the new definition of the Institute of Medicine in full.

\section{Acknowledgments}

Funding: The project is funded by the Science Research Foundation of Chinese Nursing Association (grant number: ZHKY201913) and Zhejiang University Academic Award for Outstanding Doctoral Candidates (grant number: 202059).

\section{Footnote}

Conflicts of Interest: All authors have completed the ICMJE uniform disclosure form (available at http://dx.doi. org/10.21037/apm-20-2133). The authors have no conflicts of interest to declare.

Ethical Statement: The authors are accountable for all aspects of the work in ensuring that questions related to the accuracy or integrity of any part of the work are appropriately investigated and resolved. The study was approved by The Second Affiliated Hospital Zhejiang University School of Medicine (No. 2020131) and written informed consent was obtained from all patients.

Open Access Statement: This is an Open Access article distributed in accordance with the Creative Commons Attribution-NonCommercial-NoDerivs 4.0 International License (CC BY-NC-ND 4.0), which permits the noncommercial replication and distribution of the article with the strict proviso that no changes or edits are made and the original work is properly cited (including links to both the formal publication through the relevant DOI and the license). See: https://creativecommons.org/licenses/by-nc-nd/4.0/.

\section{References}

1. Bleijlevens MH, Wagner LM, Capezuti E, et al. Physical Restraints: Consensus of a Research Definition Using a Modified Delphi Technique. J Am Geriatr Soc 2016;64:2307-10.

2. Souza LM, Santana R, Capeletto C, et al. Factors associated with mechanical restraint in the hospital environment: a cross-sectional study. Rev Esc Enferm Usp 2019;53:e03473.

3. Pun BT, Balas M, Barnes-Daly M, et al. Caring for Critically Ill Patients with the ABCDEF Bundle: Results of the ICU Liberation Collaborative in Over 15,000 Adults. Crit Care Med 2019;47:3-14.

4. Zhang C, Liu D, He Q. The characteristics of ICU physical restraint use and related influencing factors in China: a multi-center study. Ann Palliat Med 2021;10:1198-206.

5. Benbenbishty J, Adam S, Endacott R. Physical restraint use in intensive care units across Europe: the PRICE study. Intensive Crit Care Nurs 2010;26:241-5.

6. Martin B, Mathisen L. Use of physical restraints in adult critical care: a bicultural study. Am J Crit Care 2005;14:133-42.

7. Frezza EE, Carleton G, Valenziano C. A quality improvement and risk management initiative for surgical ICU patients: a study of the effects of physical restraints and sedation on the incidence of self-extubation. Am J Med Qual 2000;15:221-5.

8. Minnick AF, Mion L, Johnson M, et al. Prevalence and Variation of Physical Restraint Use in Acute Care Settings in the US. J Nurs Scholarsh 2007;39:30-7.

9. Burry L, Rose L, Ricou B. Physical restraint: time to let go. Intensive Care Med 2018;44:1296-8.

10. Devlin JW, Skrobik Y, Gelinas C, et al. Clinical Practice Guidelines for the Prevention and Management of Pain, Agitation/Sedation, Delirium, Immobility, and Sleep Disruption in Adult Patients in the ICU. Crit Care Med 2018;46:e825-73.

11. Jarachovic M, Mason M, Kerber K, et al. The role of standardized protocols in unplanned extubations in a medical intensive care unit. Am J Crit Care 2011;20:30411,312 .

12. Chang LY, Wang KW, Chao YF. Influence of physical restraint on unplanned extubation of adult intensive care patients: a case-control study. Am J Crit Care 2008;17:408$15,416$.

13. Curry K, Cobb S, Kutash M, et al. Characteristics associated with unplanned extubations in a surgical intensive care unit. Am J Crit Care 2008;17:45-51, 52.

14. Ertuğrul B, Özden D. The effect of physical restraint on neurovascular complications in intensive care units. Aust Crit Care 2020;33:30-8.

15. Liu Y, Wu X, Ma Y, et al. The prevalence, incidence, and associated factors of pressure injuries among immobile inpatients: A multicentre, cross-sectional, exploratory descriptive study in China. Int Wound J 2019;16:459-66. 
16. Rose L, Burry L, Mallick R, et al. Prevalence, risk factors, and outcomes associated with physical restraint use in mechanically ventilated adults. J Crit Care 2016;31:31-5.

17. McPherson JA, Wagner C, Boehm L, et al. Delirium in the cardiovascular ICU: exploring modifiable risk factors. Crit Care Med 2013;41:405-13.

18. Mehta S, Cook D, Devlin J, et al. Prevalence, risk factors, and outcomes of delirium in mechanically ventilated adults. Crit Care Med 2015;43:557.

19. Martin B. Restraint use in acute and critical care settings: changing practice. AACN Clin Issues 2002;13:294-306.

20. Jacob JD, Holmes D, Rioux D, et al. Convergence and divergence: An analysis of mechanical restraints. Nurs Ethics 2019;26:1009-26.

21. Registered Nurses' Association of Ontario (RNAO). Promoting safety: alternative approaches to the use of restraints. 2012. Available online: http://rnao.ca/bpg/ guidelines/promoting-safety-alternative-approaches-userestraints

22. Lach HW, Leach K, Butcher H. Evidence-Based Practice Guideline: Changing the Practice of Physical Restraint Use in Acute Care. J Gerontol Nurs 2016;42:17-26.

23. Barr J, Fraser G, Puntillo K, et al. Clinical practice guidelines for the management of pain, agitation, and delirium in adult patients in the intensive care unit. Crit Care Med 2013;41:263-306.

24. Fervers B, Burgers J, Haugh M, et al. Adaptation of clinical guidelines: literature review and proposition for a framework and procedure. Int J Qual Health Care 2006;18:167-76.

25. G-I-N Adaptation Working Group. 2010. Available online: https://g-i-n.net/working-groups/adaptation

26. Harrison M, van den Hoek J, Graham I. CANIMPLEMENT@: Planning for Best Practice Implementation. Philadelphia: Wolters Kluwer (US); 2014.

27. World Health Organization. WHO handbook for guideline development: WHO, 2014.

28. Chen Y, Ma Y, Zhou Q, et al. Who Should Participate in the Development of Clinical Practice Guidelines? Medical Journal of Peking Union Medical College Hospital (Chinese) 2019;10:524-30.

29. Whittemore R, Knafl K. The integrative review: updated methodology. J Adv Nurs 2005;52:546-53.

30. Institute of Medicine (US) Committee on Standards for Developing Trustworthy Clinical Practice Guidelines. Clinical Practice Guidelines We Can Trust. Washington (DC): National Academies Press (US); 2011.

31. Institute of Medicine (US) Committee on Standards for
Developing Trustworthy Clinical Practice Guidelines. Clinical Practice Guidelines: Directions for a New Program. Washington (DC): National Academies Press (US);1990.

32. Brouwers MC, Spithoff K, Kerkvliet K, et al. Development and Validation of a Tool to Assess the Quality of Clinical Practice Guideline Recommendations. JAMA Netw Open 2020;3:e205535.

33. Zhang M, Zhou Y, Li L, et al. The construction of content analysis and apprisal of guideline matrix table based on CAN-IMPLEMENT. Journal of Nurses Training (Chinese) 2018;33:1635-8.

34. Shea BJ, Reeves B, Wells G, et al. AMSTAR 2: a critical appraisal tool for systematic reviews that include randomised or non-randomised studies of healthcare interventions, or both. BMJ 2017;358:j4008.

35. Zhang F, Shen A, Zeng X et al. An Introduction to AMSTAR 2: a critical appraisal tool for systematic reviews. Chinese Journal of Evidence-Based Cardiovascular Medicine (Chinese) 2018;10:14-8.

36. Murphy MK, Black N, Lamping D, et al. Consensus development methods, and their use in clinical guideline development. Health Technol Assess 1998;2:i-iv.

37. Loblaw DA, Prestrud A, Somerfield M, et al. American Society of Clinical Oncology Clinical Practice Guidelines: formal systematic review-based consensus methodology. J Clin Oncol 2012;30:3136-40.

38. Wiercioch W, Akl EA, Santesso N, et al. Assessing the process and outcome of the development of practice guidelines and recommendations: PANELVIEW instrument development. CMAJ 2020;192:E1138-45.

39. Song Y, Darzi A, Ballesteros M, et al. Extending the RIGHT statement for reporting adapted practice guidelines in healthcare: the RIGHT-Ad@pt Checklist protocol. BMJ Open 2019;24;9:e031767.

40. Shekelle P, Eccles M, Grimshaw J, et al. When should clinical guidelines be updated? BMJ 2001;323:155-7.

41. Shekelle PG, Ortiz E, Rhodes S, et al. Validity of the Agency for Healthcare Research and Quality clinical practice guidelines: how quickly do guidelines become outdated? JAMA 2001;286:1461-7.

42. World Health Organization (WHO). Bridging the "Know-Do" Gap: Meeting on Knowledge Translation in Global Health. 2005. Available online: https://www.who. int/workforcealliance/knowledge/resources/knowdo_ gap/en/

43. International Council of Nurses (ICN). Closing the gap: From evidence to action. 2012. Available online: http:// 
www.icn.ch/publications/2012-closing-the-gap-fromevidence-to-action/

44. Lockwood C, Lizarondo L, Harrison M, et al. Initial perceptions of, and intention to use, an online guideline adaptation framework: a descriptive survey. Int J Evid Based Healthc 2018;16:214-26.

45. Langley G, Schmollgruber S, Egan A. Restraints in intensive care units--a mixed method study. Intensive Crit Care Nurs 2011;27:67-75.

46. Cui N, Long M, Zhou S, et al. Knowledge, Attitudes, and Practices of Chinese Critical Care Nurses Regarding Physical Restraint. J Contin Educ Nurs 2019;50:121-6.

(English Language Editors: B. Draper and J. Chapnick)

Cite this article as: Cui N, Zhang Y, Liu Y, Zhou Y, Sun H, Jin $\mathrm{J}$; on behalf of the guideline adaptation working group of physical restraints. Protocol for the adaptation of clinical practice guidelines for the management of physical restraints in critically ill patients. Ann Palliat Med 2021;10(4):4889-4896. doi: 10.21037/apm-20-2133 
Supplementary

Table S1 Evidence Search

\begin{tabular}{|c|c|}
\hline Category & Name and Link \\
\hline Databases & $\begin{array}{l}\text { - Guidelines International Network (GIN): https://g-i-n.net/ } \\
\text { - } \text { Scottish Intercollegiate Guidelines Network (SIGN): https://www.sign.ac.uk/ } \\
\text { - } \text { Institute for Clinical Systems Improvement (ICSI): https://www.icsi.org/guidelines/ } \\
\text { - Registered Nurses' Association of Ontario (RNAO): https://rnao.ca/ } \\
\text { - Canadian Task Force on Preventive Healthcare (CTFPHC): https://www.canadiantaskforce.ca/ } \\
\text { - Joanna Briggs Institute (JBI) } \\
\text { - The Cochrane library } \\
\text { - EMBASE } \\
\text { - PubMed } \\
\text { - Web of Science } \\
\text { - Cumulative Index to Nursing and Allied Health Literature (CINAHL) } \\
\text { - Psyclnfo } \\
\text { - Psyc Articles } \\
\text { - Psychology and Behavioral Science Collection } \\
\text { - China National Knowledge Infrastructure (CNKI, For Chinese) } \\
\text { - SinoMed (For Chinese) } \\
\text { - WANFANG DATA (For Chinese) }\end{array}$ \\
\hline Professional associations & $\begin{array}{l}\text { - World Federation of Critical Care Nurses (WFCCN): https://wfccn.org/clinical-guidelines/ } \\
\text { - Society of Critical Care Medicine (SCCM): https://www.sccm.org/Research/Guidelines } \\
\text { - } \text { European Society of Intensive Care Medicine (ESICM): https://www.esicm.org/resources/guidelines- } \\
\text { consensus-statements/ } \\
\text { - British Association of Critical Care Nurses (BACCN): https://www.baccn.org/ } \\
\text { - New Zealand Nurses Organization (NZNO): https://www.nzno.org.nz/resources/medicines_-- } \\
\text { guidelines_and_information } \\
\text { - Chinese Nursing Association (CNA): http://www.zhhlxh.org.cn/cnaWebcn/ } \\
\text { - The Hong Kong Society of Critical Care Medicine (HKSCCM): https://www.hksccm.org/index.php/ } \\
\text { professional/useful-resources }\end{array}$ \\
\hline
\end{tabular}


PubMed \#1 Restraint, Physical [MeSH Major Topic]

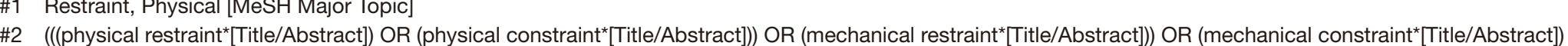

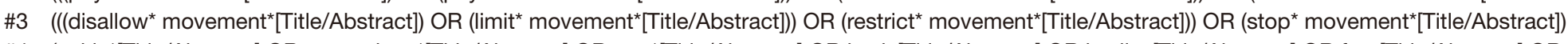

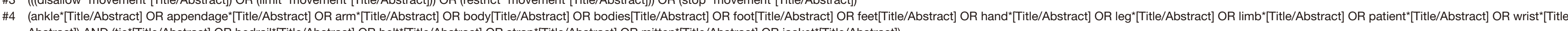

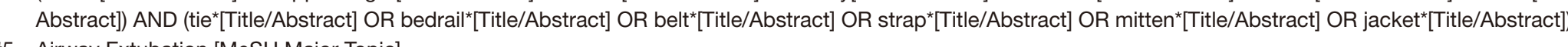

\#5 Airway Extubation [MeSH Major Topic]

\#7 Respiration, Artificial [MeSH Major Topic

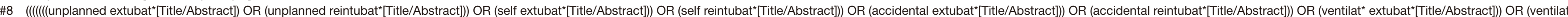

reintubat*Title/Abstract)

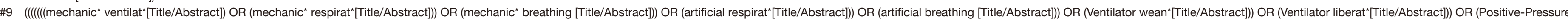
Respiration [Titte/Abstract])

\#10 Aggression [MeSH Major Topic]

Hit

\#13 Behavior Control [MeSH Major Topic]

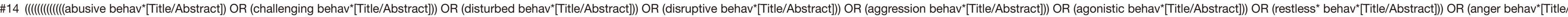
Abstract])) OR (angry behav"[Tite/Abstract])) OR (assault behav"(Title/Abstract])) OR (rage* behav"

$\# 15$ (daily sedation interruption [Title/Abstract)) OR (crisis intervention [Title/Abstract])

$\# 16$ Delirium [MeSH Major Topic]

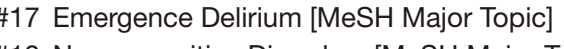

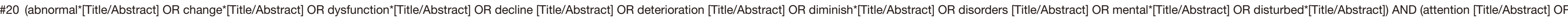
cognitive [Title/Abstract] OR consciousness [Title/Abstract] OR perception [Titte/Abstract] OR neurocognitive [Titte/Abstract])

\#21 (acute brain syndrome [Titte/Abstract) OR (acute cerebral insufficiency [Title/Abstract])

\#22 Pain, Procedural [MeSH Major Topic]

\#23 Pain, Postoperative [MeSH Major Topic]

\#25 Pain Measurement [MSSH Major Topic]

\#26 Analgesics, Non-Narcotic [MeSH Major Topic]

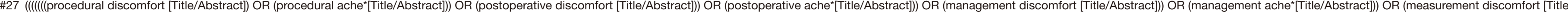
Abstract])) OR (measurement ache*[Title/Abstract)

\#28 Decision Making, Shared [MeSH Major Topic]

\#29 Family Separation [MeSH Major Topic Tor

(open visiting [Title/Abstract])) OR (flexible visitation [Titte/Abstract])) OR (flexible visiting [Titte/Abstract])) OR (restrictive visitation [Titte/Abstract])) OR (restrictive visiting [Title/Abstract])) OR (family visitation [Title/Abstract])) OR (family visiting [Title/Abstract)

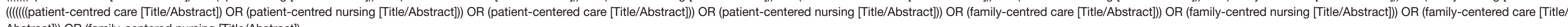

Major Topic]

\#34 Critical Care [MeSH Major Topic]

\#35 Critical Care Nursing [MeSH Major Topic]

cal care [Title/Abstract)) OR (acute care [Title/Abstract])

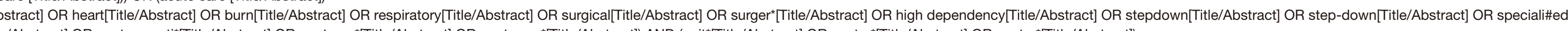

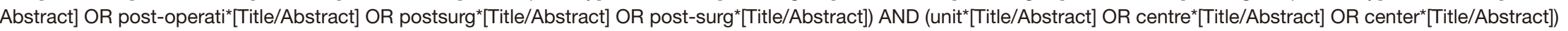

$\# 38$ ICU*Titile//ASH

$\# 40$ OR \#33-\#38

\#41 \#39 AND \#40

H2 Filters: Guideline, Practice Guideline, Humans, Chinese, English, from 2011-2020 
Table S3 Guideline content analysis and assessment matrix

Guideline content analysis and assessment matrix

Basic information

Title of the Guideline

Publication Associations

Publication Year

Type of the Guideline

Methodology of Guideline Development

Criteria for Quality of Evidence and Strength of Recommendations

The Timeliness of Evidence

Search time

The comprehensiveness of Search

Quality of the Guideline

Appraisal of Guidelines for Research \& Evaluation II Domain 1. Scope and Purpose (AGREE II)

Domain 2. Stakeholder Involvement

Domain 3. Rigour of Development

Domain 4. Clarity of Presentation

Domain 5. Applicability

Domain 6. Editorial Independence

Overall Guideline Assessment

$\square$ Recommend $\square$ Recommend with Modifications $\square$ Not Recommend

Quality of Guideline Recommendations

Clinical Questions

Recommendations

Grades of Recommendation

Appraisal of Guidelines Research and EvaluationRecommendations Excellence (AGREE-REX)

Domain 1. Clinical Applicability

Domain 2. Values and Preferences

Domain 3. Implementability

Overall Assessment Statements $\square$ Recommend $\square$ Recommend with Modifications $\square$ Not Recommend 
Table S4 Systematic review/meta-analysis content analysis and assessment matrix

\begin{tabular}{llllll}
\hline Title & Studies Included (participants) & Interventions & Control & AMSTAR2 Rating & Outcomes \\
\hline
\end{tabular}

AMSTAR2: A Measurement Tool to Assess Systematic Reviews; GRADE: Grading of Recommendations Assessment, Development and Evaluation

Table S5 Recommendations matrix

\begin{tabular}{|c|c|c|c|c|c|c|}
\hline \multicolumn{7}{|l|}{ Recommendations matrix } \\
\hline \multicolumn{7}{|l|}{ Outline Specific Recommendation } \\
\hline \multicolumn{7}{|l|}{ Source of Evidence } \\
\hline \multicolumn{7}{|l|}{ Quality of Evidence } \\
\hline \multicolumn{7}{|l|}{ Patient Values and Preferences } \\
\hline \multicolumn{7}{|l|}{ Acceptability of Policy-makers } \\
\hline \multicolumn{7}{|l|}{ Judgement } \\
\hline Benefits\&Harms & $\begin{array}{l}\text { Quality of } \\
\text { Evidence }\end{array}$ & $\begin{array}{l}\text { Resources } \\
\text { Required }\end{array}$ & Equity & Feasibility & $\begin{array}{l}\text { Values and } \\
\text { Preferences }\end{array}$ & Acceptability \\
\hline$\square$ Benefits more than harms & & $\begin{array}{l}\square \text { Moderate saving } \\
\square \text { Large saving }\end{array}$ & 口Increased & & & $\square$ Yes \\
\hline Strength of Recommendation & & \multirow{2}{*}{\multicolumn{2}{|c|}{$\square$ Strong Recommendation }} & \multicolumn{3}{|c|}{$\square$ Conditional Recommendation } \\
\hline \multicolumn{5}{|l|}{ The Feedback and Changes } & & \\
\hline
\end{tabular}

